\title{
Noise-Figure Measurement of Differential Amplifiers Using Nonideal Baluns
}

\author{
Óscar García-Pérez, Vicente González-Posadas, Luis Enrique García-Muñoz, and \\ Daniel Segovia-Vargas, Member, IEEE
}

\begin{abstract}
This paper analyzes the noise and gain measurement of microwave differential amplifiers using two passive baluns. A general model of the baluns is considered, including potential losses and phase/amplitude unbalances. This analysis allows de-embedding the actual gain and noise performance of the isolated amplifier by using single-ended measurements of the cascaded system and baluns. Finally, measured results from two amplifier prototypes are used to validate the theoretical principles.
\end{abstract}

Index Terms-Baluns, differential amplifiers, low-noise amplifiers, noise measurement.

\section{INTRODUCTION}

D URING THE last decades, differential amplifiers have been widely used in low-frequency electronics. More recently, engineers have exploited the interesting properties of differential amplifiers in the microwave range to improve the interference mitigation or the dynamic range. One of the applications in which differential amplifiers are being used is the design of differential active antenna arrays for the next generation of radio telescopes (e.g., Square Kilometre Array) [1]. The use of a differential active device directly connected to a differential Vivaldi antenna element avoids the use of a passive balun, and therefore, allows improving the system noise temperature. For these types of applications, a method to accurately characterize the gain and noise performance of the low-noise amplifiers for non-50- $\Omega$ antenna impedances is required. Nevertheless, the main constraint with differential devices is their nontrivial characterization at microwave frequencies [2].

Mixed-mode scattering parameters ( $S$-parameters) are defined in order to facilitate the analysis of differential devices since they make use of differential or common-mode excitations instead of conventional single-ended ones [3]. These parameters can be calculated from the conventional $S$-parameter matrix [3] or directly measured with a multiport network analyzer [4].

However, although $S$-parameter characterization of differential devices is possible, the noise measurement is still a challenge at microwave frequencies. A method based on a hot/cold differential load connected at the input of the amplifier has been presented in [5]. This method requires a mechanism to cool the load (e.g., liquid $\mathrm{N}_{2}$ ) that may not be easily available. Although resistive load impedances can be easily used, arbitrary complex source impedances can be difficult to synthesize. Furthermore, the connecting lines between the load and input of the amplifier introduce a phase rotation that distorts the impedance connected at the edge. In [6], another method to determine the noise figure of a differential amplifier has been described. It is based on measuring the partial gain and noise between pairs of input/output ports. Although it only requires a conventional gain and noise analyzer for 50- $\Omega$ measurements, the limitation of this method is that it is difficult to synthesize arbitrary and symmetrical source impedances for the input differential ports. This fact would require a special differential impedance tuner [7].

The use of passive baluns is the simplest method to characterize differential amplifiers. Any source impedance condition can be generated by using a conventional single-ended impedance tuner, which is indispensable to obtain the noise circles of the differential device-under-test (DUT) [7]. Since the external baluns affect the measurement procedure, it is necessary to de-embed the gain and noise values of the isolated DUT from the measurement of the cascaded system (i.e., input balun, amplifier, and output balun). However, the de-embedding methods described in the literature [8] assume a simplified model of the balun, with symmetrical losses and ideal $180^{\circ}$ phase difference between the output ports, which is far from the behavior of real baluns used in practice. With this simplified balun model, only differential-mode signals are propagated so the effect of common-mode signals was not taken into account. Thus, there is not a procedure to de-embed the noise performance of the DUT when phase unbalances or asymmetrical amplitude responses appear in the baluns.

This paper analyzes the noise performance of differential amplifiers with input/output baluns using a general model of the baluns with arbitrary losses and phase/amplitude unbalances in both branches. The main improvement with this analysis is that it considers the presence of both differential and common-mode signals due to the unbalances in the balun, and therefore, provides a more accurate measurement. The proposed procedure can be used for both 50- $\Omega$ measurements and for source-pull measurements using an additional conventional load-pull tuner at the input [7].

\section{THEORETICAL ANALYSIS}

The method that will be analyzed in this section is shown in Fig. 1(a). Ideally, the input balun equally splits the signal 


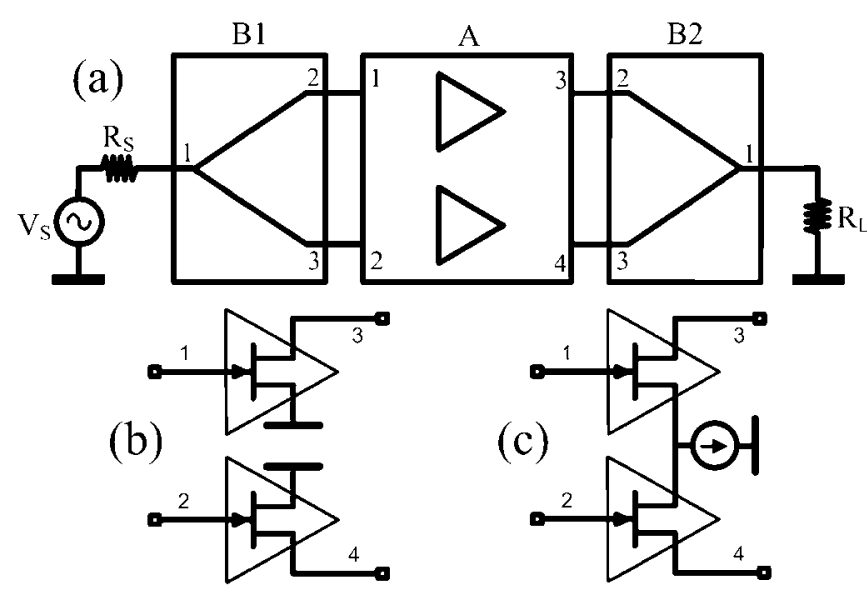

Fig. 1. (a) Measurement procedure of a differential amplifier (A) using baluns (B1 and B2) and typical circuit schematics of a: (b) balanced and (c) fully differential amplifier.

along two $180^{\circ}$ out-of phase output branches. The behavior of the output balun is equivalent, although combining the output signals from the amplifiers. Thus, the measured noise figure of the system with ideal baluns is equal to the noise figure of the isolated amplifier with differential excitations [8].

Differential amplifiers can be classified into two groups: balanced and fully differential [see Fig. 1(b) and (c)] [2]. Although the response when using differential excitations is equivalent in both cases, it is not so for common-mode excitations. Balanced amplifiers equally amplify both differential- and common-mode signals, which means CMRR $=1$ [common-mode rejection ratio (CMRR)]. However, fully differential amplifiers only amplify differential signals since common-mode excitations are mitigated by the structure (i.e., CMRR $=\infty$ ). If a simplified model of the balun with symmetrical losses and no phase unbalance were used, only differential signals would propagate along the system, and a single general model of the amplifier could be considered [8]. In this case, both losses and phase/magnitude unbalances are considered in the baluns. Therefore, since both differential- and common-mode signals may appear, a separate analysis for the balanced and fully differential amplifier should be done.

\section{A. Balanced Amplifier}

Balanced amplifiers consist of two independent amplifiers working in parallel [see Fig. 1(b)]. In this case, all the $S$-parameters of the amplifier are assumed to be zero, except the transmission parameters between ports $1-3$ and $2-4$, i.e.,

$$
s_{31}^{A}=s_{42}^{A}=A
$$

where $A$ is the gain factor of the amplifier. For the case of the baluns, it is assumed that all the $S$-parameters are zero (perfect impedance matching and output ports isolation), except the transmission coefficients between ports $1-2$ and 1-3 (arbitrary losses and phase/magnitude unbalances), i.e.,

$$
\begin{array}{ll}
s_{12}^{B 1}=s_{21}^{B 1} & s_{13}^{B 1}=s_{31}^{B 1} \\
s_{12}^{B 2}=s_{21}^{B 2} & s_{13}^{B 2}=s_{31}^{B 2}
\end{array}
$$

where super-indices $B 1$ and $B 2$ indicate the input and output baluns, respectively, assuming perfect output ports isolation and impedance matching in the balun is necessary to ensure that the amplifier loading conditions are the same as in the case of a $50-\Omega$ isolated environment. Otherwise, the amplifier would not be correctly excited and the de-embedding would not be feasible. For the case of the $S$-parameters, one can re-normalize the parameters matrix obtained for certain port impedance conditions and extract the corresponding matrix for any other port impedance conditions. However, this is not so for noise measurements. Measuring the noise figure (even knowing the $S$-parameters) of a device under certain port-impedance conditions does not give enough information to analytically obtain the corresponding noise figure for other matching conditions. It would require to have available the complete set of noise parameters (i.e., minimum noise figure $\left(F_{\min }\right)$, optimum noise matching $\left(\Gamma_{\text {opt }}\right)$, and noise resistance $\left(R_{n}\right)$ ), which is assumed to be unknown a priori in this case. This last point could be achieved by exciting the amplifier for many source impedances (i.e., by using an additional impedance tuner) and following the method in Fig. 1, as was explained in [7].

The noise factor of a two-port device can be written as

$$
f=\frac{\text { Total noise power at the output }}{\text { Noise power at the output due to the source resistor }} \text {. }
$$

In this case, the noise power at the output port is due to the contributions from the source resistor, from the two baluns, and from the amplifier. For the input balun, it can be assumed that the two branches generate uncorrelated noise signals [8]. Since the noise factor of a passive device is equal to its losses, the noise spectral density at its output is $k T\left(\mathrm{~W}^{2} / \mathrm{Hz}\right)$, where $k$ is the Boltzmann constant and $T$ is the system temperature. For the case of the 1-2 and 1-3 branches of the input balun, this power density propagates to the output through the amplifier and the output balun as

$$
\begin{aligned}
& N_{1-2}^{B 1, a}=k T\left|s_{31}^{A} s_{12}^{B 2}\right|^{2} \\
& N_{1-3}^{B 1, a}=k T\left|s_{42}^{A} s_{13}^{B 2}\right|^{2}
\end{aligned}
$$

where sub-indices 1-2 and 1-3 indicate the corresponding branch of the balun; super index $a$ is used for the balanced topology, while super-indices $B 1, A, B 2$ denote the corresponding input balun or amplifier or output balun parameter. Terms in (4) contain the combined contribution of the source resistor and the input balun. For the balanced amplifier, it can be assumed that the noise generated by the two independent branches is uncorrelated. By definition, the noise power at the output of an amplifier is $k T f_{A} G_{A}$, where $f_{A}$ is the noise factor of the amplifier and $G_{A}$ is the power gain [8]. In order to calculate the contribution of the amplifier alone, the contribution from the source must be eliminated (i.e., $k T G_{A}$ ). Thus, the noise power of each stage of the balanced amplifier propagated to the output of the system is, respectively,

$$
\begin{aligned}
N_{1}^{A, a} & =\left(k T f_{A}\left|s_{31}^{A}\right|^{2}-k T\left|s_{31}^{A}\right|^{2}\right)\left|s_{12}^{B 2}\right|^{2} \\
& =k T\left(f_{A}-1\right)\left|s_{31}^{A} s_{12}^{B 2}\right|^{2} \\
N_{2}^{A, a} & =k T\left(f_{A}-1\right)\left|s_{42}^{A} s_{13}^{B 2}\right|^{2}
\end{aligned}
$$


where sub-indices 1 and 2 indicate the corresponding input port of the amplifier. The noise spectral density at the output of the balun B2 is $k T\left(\mathrm{~W}^{2} / \mathrm{Hz}\right)$. Since two independent source resistors launch noise at the second and third output balun ports, the noise generated by the balun alone can be obtained as

$$
\begin{aligned}
N^{B 2, a} & =k T-k T\left|s_{12}^{B 2}\right|^{2}-k T\left|s_{13}^{B 2}\right|^{2} \\
& =k T\left[1-\left(\left|s_{12}^{B 2}\right|^{2}+\left|s_{13}^{B 2}\right|^{2}\right)\right] .
\end{aligned}
$$

Finally, the noise contribution from the source resistor at the output of the system is obtained as

$$
N^{S, a}=k T\left|s_{21}^{B 1} s_{31}^{A} s_{12}^{B 2}+s_{31}^{B 1} s_{42}^{A} s_{13}^{B 2}\right|^{2} .
$$

From the last expression, the power gain of the cascaded system when using a balanced amplifier can be extracted, i.e.,

$$
G_{\text {casc }}^{(a)}=\left|s_{21}^{B 1} s_{31}^{A} s_{12}^{B 2}+s_{31}^{B 1} s_{42}^{A} s_{13}^{B 2}\right|^{2} .
$$

The noise figure of the cascaded system can be obtained from (3)-(7) as

$$
\begin{aligned}
& f_{\text {casc }}^{(a)} \\
& =\frac{N_{1-2}^{B 1, a}+N_{1-3}^{B 1, a}+N_{1}^{A, a}+N_{2}^{A, a}+N^{B 2, a}}{N^{S, a}} \\
& =\frac{f_{A}\left(\left|s_{31}^{A} s_{12}^{B 2}\right|^{2}+\left|s_{42}^{A} s_{13}^{B 2}\right|^{2}\right)+1-\left(\left|s_{12}^{B 2}\right|^{2}+\left|s_{13}^{B 2}\right|^{2}\right)}{\left|s_{21}^{B 1} s_{31}^{A} s_{12}^{B 2}+s_{31}^{B 1} s_{42}^{A} s_{13}^{B 2}\right|^{2}} .
\end{aligned}
$$

If the mixed-mode parameters of the baluns were used instead of the conventional ones [2], i.e.,

$$
\begin{aligned}
& s_{d s 21}^{B 1}=\left(s_{21}^{B 1}-s_{31}^{B 1}\right) / \sqrt{2} \\
& s_{c s 21}^{B 1}=\left(s_{21}^{B 1}+s_{31}^{B 1}\right) / \sqrt{2} \\
& s_{s d 21}^{B 2}=\left(s_{12}^{B 2}-s_{13}^{B 2}\right) / \sqrt{2} \\
& s_{s c 21}^{B 2}=\left(s_{12}^{B 2}+s_{13}^{B 2}\right) / \sqrt{2}
\end{aligned}
$$

where the sub-indices $c, d$, and $s$ denote differential-, common-, and single-ended excitations, respectively. In addition, the subindex order in the mixed-mode parameters considers the differential or common-mode outputs for the first balun and singleended output for the second balun. Assuming an ideal balanced amplifier (1) and using mixed-mode $S$-parameter notation (10), (9) can then be simplified to

$$
f_{\text {casc }}^{(a)}=\frac{\left(f_{A}|A|^{2}-1\right)\left(\left|s_{s d 21}^{B 2}\right|^{2}+\left|s_{s c 21}^{B 2}\right|^{2}\right)+1}{\left|\left(s_{d s 21}^{B 1} A s_{s d 21}^{B 2}+s_{c s 21}^{B 1} A s_{s c 21}^{B 2}\right)\right|^{2}} .
$$

It can be seen that for the case of ideal baluns, i.e.,

$$
\begin{array}{ll}
s_{21}^{B 1}=1 / \sqrt{2} & s_{31}^{B 1}=-1 / \sqrt{2} \\
s_{12}^{B 2}=1 / \sqrt{2} & s_{13}^{B 2}=-1 / \sqrt{2}
\end{array}
$$

the noise figure and gain of the cascaded system is equal to the performance of the isolated amplifier, i.e.,

$$
f_{\text {casc }}=f_{A} \text { and } G_{\text {casc }}=A \text {. }
$$

\section{B. Fully Differential Amplifier}

A fully differential amplifier, also known as a differential pair, is an amplifier in which the sources of two transistors are both connected to a common current source [see Fig. 1(c)]. Unlike the previous case, the differential pair cannot be analyzed as two independent stages. Thus, in the ideal case, all the $S$-parameters of the amplifier are zero, except the transmission parameters between the input ports and the output ports, i.e.,

$$
\begin{gathered}
s_{31}^{A}=s_{42}^{A}=A / 2 \\
s_{32}^{A}=s_{41}^{A}=-A / 2 .
\end{gathered}
$$

In this case, the noise power present in one of the inputs of the amplifier is propagated through the two outputs. Thus, the noise coming from one branch of the input balun is divided in two paths in the amplifier and combined in the output balun as

$$
\begin{aligned}
& N_{1-2}^{B 1, b}=k T\left|s_{31}^{A} s_{12}^{B 2}+s_{41}^{A} s_{13}^{B 2}\right|^{2} \\
& N_{1-3}^{B 1, b}=k T\left|s_{32}^{A} s_{12}^{B 2}+s_{42}^{A} s_{13}^{B 2}\right|^{2}
\end{aligned}
$$

where sub-indices 1-2 and 1-3 indicate the corresponding branch of the balun. The noise contribution of the differential amplifier can be calculated assuming two independent noise sources in both input ports. Therefore, the noise launched by each source is propagated through the two outputs of the amplifier and finally combined by the output balun as

$$
\begin{aligned}
& N_{1}^{A, b}=k T\left(f_{A}-1\right)\left|s_{31}^{A} s_{12}^{B 2}+s_{41}^{A} s_{13}^{B 2}\right|^{2} \\
& N_{2}^{A, b}=k T\left(f_{A}-1\right)\left|s_{32}^{A} s_{12}^{B 2}+s_{42}^{A} s_{13}^{B 2}\right|^{2}
\end{aligned}
$$

where sub-indices 1 and 2 indicate the corresponding input port of the amplifier. For the output balun, the noise coming from ports 2 and 3 cannot be considered uncorrelated in this case since there are not two independent paths in the overall scheme. As the differential pair only propagates differential signals (common-mode gain is zero), the noise can be modeled as a differential source connected between ports 2 and 3 . The output balun can then be interpreted as a two-port passive device, with a differential input port and a single-ended output port, whose transmission parameter $s_{s d 21}$ can be obtained from (10). Since the output noise power density is $k T\left(\mathrm{~W}^{2} / \mathrm{Hz}\right)$, the contribution of the balun alone is obtained as

$$
N^{B 2, b}=k T\left(1-0.5\left|s_{12}^{B 2}-s_{13}^{B 2}\right|^{2}\right) .
$$

Finally, the noise contribution from the source resistor propagated to the output can be calculated from the combination of the different paths along which the source signal propagates, i.e.,

$$
\begin{aligned}
N^{S, b}=k T \mid\left(s_{21}^{B 1} s_{31}^{A}+s_{31}^{B 1} s_{32}^{A}\right) s_{12}^{B 2} & \\
& +\left.\left(s_{21}^{B 1} s_{41}^{A}+s_{31}^{B 1} s_{42}^{A}\right) s_{13}^{B 2}\right|^{2} .
\end{aligned}
$$

The gain of the cascaded system in this case is directly obtained from (18) as

$$
\begin{aligned}
G_{\mathrm{casc}}^{(b)}=\mid\left(s_{21}^{B 1} s_{31}^{A}+s_{31}^{B 1} s_{32}^{A}\right) & s_{12}^{B 2} \\
& +\left.\left(s_{21}^{B 1} s_{41}^{A}+s_{31}^{B 1} s_{42}^{A}\right) s_{13}^{B 2}\right|^{2}
\end{aligned}
$$


and the corresponding noise figure is shown in (20) at the bottom of this page. For an ideal fully differential amplifier (14) and using notation of mixed-mode $S$-parameters for the baluns, (10), (20) can be simplified to

$$
f_{\text {casc }}^{(b)}=\frac{\left(f_{A}|A|^{2}-1\right)\left|s_{s d 21}^{B 2}\right|^{2}+1}{\left|s_{d s 21}^{B 1} A s_{s d 21}^{B 2}\right|^{2}} .
$$

It can be seen that (21) is analogous to (11), but removing the common-mode terms. That conclusion is logical since the fully differential amplifiers mitigate the common-mode signals.

\section{Step-by-Step Gain and Noise Measurement Procedure}

The step-by-step gain and noise measurement procedure for both balanced and fully differential amplifier topologies is summarized below.

1) Characterization of the balun.

Measure the transmission parameters (2) of baluns B1 and $\mathrm{B} 2$ by using a conventional network analyzer. Also, it should be checked that the reflection coefficients and the output ports isolations are significantly lower (e.g., lower than $-15 \mathrm{~dB}$ ) in the band of interest.

2) Gain measurement.

Connect the baluns and the differential amplifier following the scheme shown in Fig. 1(a). Measure the gain $G_{\text {casc }}$ of the two-port cascaded system by means of a conventional two-port network or gain analyzer. Assuming condition (1) in the case of the balanced amplifier or (14) in the case of the fully differential amplifier, de-embed the gain $A$ of the amplifier making use of (8) or (19), respectively.

3) Noise measurement.

Measure the noise figure $f_{\text {casc }}$ of the cascaded system by using a conventional two-port noise analyzer. Making use of (9) in the case of the balanced amplifier or (20) in the case of the fully differential amplifier, de-embed the noise figure $f_{A}$ of the amplifier.

\section{Numerical Example: De-Embedding the Noise Figure}

Equations (9) and (20) give analytical expressions that can be used to de-embed the noise figure of a balanced or a fully differential amplifier using nonideal baluns. Nevertheless, the choice of a proper configuration of a given set of baluns can diminish the noise increment in $f_{\text {casc }}$, and therefore, reduce the uncertainty and errors during the de-embedding due to the limitations of the measurement equipments. A numerical example has been computed by using the formulas presented in the previous section, in order to illustrate the effects of the baluns over the measurement of a balanced and a fully differential amplifier. For both cases, it is assumed an amplifier with a gain $A=6 \mathrm{~dB}$, and a noise figure $f_{A}=1 \mathrm{~dB}$. Additionally, the same examples

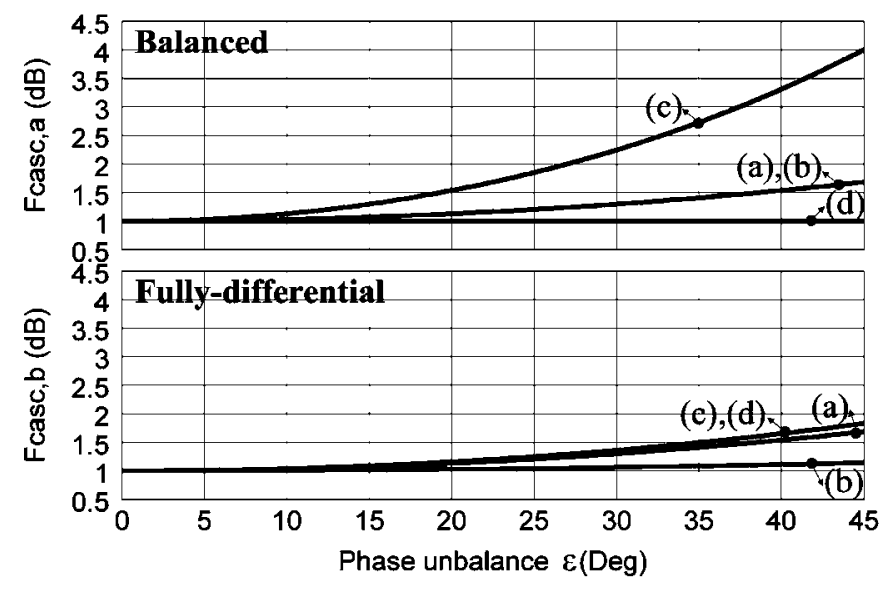

Fig. 2. Phase unbalance effect in different scenarios. (a) Unbalance in B1 $\left(\varepsilon_{\mathrm{B} 1}=\varepsilon\right)$. (b) Unbalance in $\mathrm{B} 2\left(\varepsilon_{\mathrm{B} 2}=\varepsilon\right)$. (c) Unbalances in phase in $\mathrm{B} 1$ and $\mathrm{B} 2\left(\varepsilon_{\mathrm{B} 1}=\varepsilon_{\mathrm{B} 2}=\varepsilon\right)$. (d) Unbalances in antiphase in $\mathrm{B} 1$ and $\mathrm{B} 2\left(\varepsilon_{\mathrm{B} 1}=\varepsilon\right.$, $\left.\varepsilon_{\mathrm{B} 2}=-\varepsilon\right)$.

have been validated by means of the software AWR Microwave Office obtaining the same results.

Fig. 2 shows the effect of the phase unbalances in the baluns. It should be noticed that phase unbalances are not treated in the de-embedding analysis developed in the literature [8]. The unbalances $\varepsilon_{B 1}$ and $\varepsilon_{B 2}$ are defined as the increment in the phase difference between the output ports of both baluns with respect to the ideal $180^{\circ}$, i.e.,

$$
\begin{aligned}
& \angle s_{21}^{B 1}-\angle s_{31}^{B 1}=180^{\circ}+\varepsilon_{B 1} \\
& \angle s_{12}^{B 2}-\angle s_{13}^{B 2}=180^{\circ}+\varepsilon_{B 2} .
\end{aligned}
$$

Several scenarios have been simulated, with unbalances in one or both baluns, and assuming that they do not present losses (see legend of Fig. 2). The first scenario, (a), only considers a phase unbalance in the input balun B1 $\left(\varepsilon_{\mathrm{B} 1}=\varepsilon\right)$; the second scenario, (b), only considers a phase unbalance in the output balun $\left(\varepsilon_{\mathrm{B} 2}=\varepsilon\right)$; the third scenario, (c), considers equal phase unbalances in the input and output balun $\left(\varepsilon_{\mathrm{B} 1}=\varepsilon_{\mathrm{B} 2}=\varepsilon\right)$; finally, the fourth scenario, (d), considers equal, but opposite, phase unbalance in both the input and output baluns $\left(\varepsilon_{\mathrm{B} 1}=\varepsilon, \varepsilon_{\mathrm{B} 2}=-\varepsilon\right)$. For the case of the balanced amplifier, scenarios (a) and (b) (the same unbalanced balun connected at the input or the output) equally affects in the measurement. For the third scenario, (c), the increase in the noise figure for the balanced configuration is the largest since there is no compensation between the input and output baluns. However, for the fourth scenario, (d), there is no increment in the noise figure since the unbalances of B1 and B2 compensate each other. Thus, for the balanced configuration, if two identical baluns are used to characterize a balanced amplifier, it is a good practice to connect them antisymmetrically (port 2 of $\mathrm{B} 1$ and port 3 of $\mathrm{B} 2$ in the upper path and vice versa). For

$$
f_{\text {casc }}^{(b)}=\frac{N_{1-2}^{B 1, b}+N_{1-3}^{B 1, b}+N_{1}^{A, b}+N_{2}^{A, b}+N^{B 2, b}}{N^{S, b}}=\frac{f_{A}\left(\left|s_{31}^{A} s_{12}^{B 2}+s_{41}^{A} s_{13}^{B 2}\right|^{2}+\left|s_{32}^{A} s_{12}^{B 2}+s_{42}^{A} s_{13}^{B 2}\right|^{2}\right)+1-0.5\left|s_{12}^{B 2}-s_{13}^{B 2}\right|^{2}}{\left|\left(s_{21}^{B 1} s_{31}^{A}+s_{31}^{B 1} s_{32}^{A}\right) s_{12}^{B 2}+\left(s_{21}^{B 1} s_{41}^{A}+s_{31}^{B 1} s_{42}^{A}\right) s_{13}^{B 2}\right|^{2}}
$$




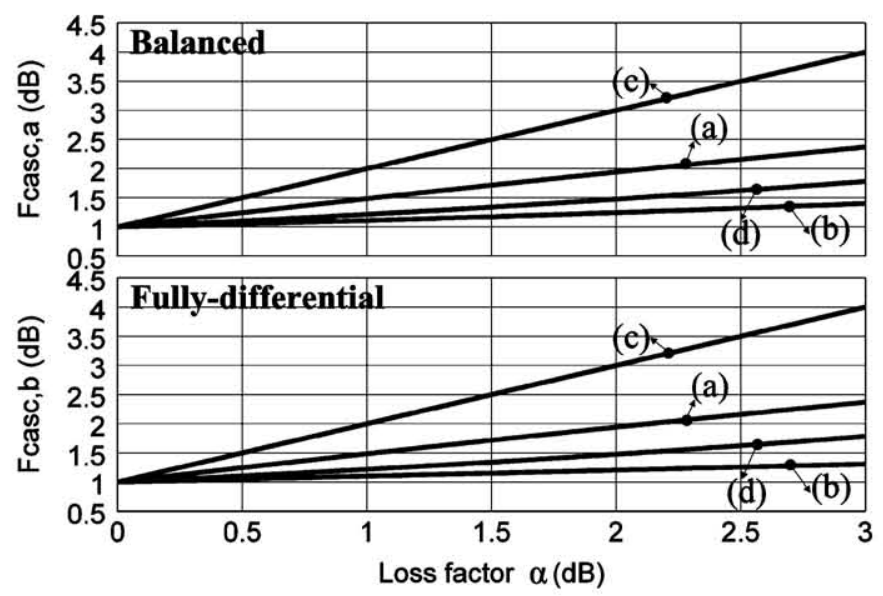

Fig. 3. Losses effect in different scenarios. (a) Unbalance only in B1 $\left(\alpha_{\mathrm{B} 1}^{\prime}=\right.$ $\left.\alpha, \alpha_{\mathrm{B} 1}^{\prime \prime}=1\right)$. (b) Unbalance only in $\mathrm{B} 2\left(\alpha_{\mathrm{B} 2}^{\prime}=\alpha, \alpha_{\mathrm{B} 2}^{\prime \prime}=1\right)$. (c) Symmetric losses only in B1 $\left(\alpha_{\mathrm{B} 1}^{\prime}=\alpha_{\mathrm{B} 1}^{\prime \prime}=\alpha\right)$. (d) Symmetric losses only in $\mathrm{B} 2\left(\alpha_{\mathrm{B} 2}^{\prime}=\right.$ $\left.\alpha_{\mathrm{B} 2}^{\prime \prime}=\alpha\right)$.

the case of the fully differential amplifier, the effect of the input balun is the same as the one with the balanced amplifier, (a), but the effect of the output balun is mitigated by the gain factor of the amplifier, (b). For the case of unbalances in the two baluns, it is indifferent whether the unbalances appear in phase or antiphase, [(c) or (d)].

The losses and amplitude unbalance effects are now analyzed. Fig. 3 shows the impact of the losses and magnitude unbalances of the balun in the noise-figure measurement. The loss factors are defined from

$$
\begin{array}{ll}
\left|s_{21}^{B 1}\right|=1 /\left(\alpha_{B 1}^{\prime} \sqrt{2}\right) & \left|s_{31}^{B 1}\right|=1 /\left(\alpha_{B 1}^{\prime \prime} \sqrt{2}\right) \\
\left|s_{12}^{B 2}\right|=1 /\left(\alpha_{B 2}^{\prime} \sqrt{2}\right) & \left|s_{13}^{B 2}\right|=1 /\left(\alpha_{B 2}^{\prime \prime} \sqrt{2}\right) .
\end{array}
$$

It can be seen that the effect is very similar for the two types of amplifier. As is expected from the Friis formula, the losses in the output balun [(b) and (d)] are less critical than the losses in the input balun [(a) and (c)] since they are mitigated by the gain factor of the amplifier. It should be noticed that [8] only considers symmetrical losses in the baluns (i.e., scenarios (c) and (d) in this case), but does not explain how to deal with the amplitude unbalances [i.e., scenarios (a) and (b)].

\section{EXPERIMENTAL RESULTS}

This section shows the experimental measurements obtained with two differential amplifier prototypes and two passive baluns. Fig. 4 shows a photograph of the manufactured prototypes. The baluns have been implemented using the well-known topology of a rat-race coupler (loading the isolated port with $50 \Omega$ ), which intrinsically gives half-power splitting with $180^{\circ}$ phase difference. The circuit schematic of the baluns is shown in Fig. 5(c). The center frequency of the baluns is $2100 \mathrm{MHz}$. Fig. 6 shows the measured transmission parameters and phase unbalance of both baluns. It should be noticed that the phase unbalance is computed as the difference with respect to the ideal $180^{\circ}$ phase difference. The frequency range between $1600-2600 \mathrm{MHz}$ is the bandwidth in which all the reflection coefficients $\left(\mathrm{s}_{11}, \mathrm{~s}_{22}\right.$, and $\left.\mathrm{s}_{33}\right)$ and the isolation parameters $\left(\mathrm{s}_{23}, \mathrm{~s}_{32}\right)$ of the baluns are lower than $-15 \mathrm{~dB}$, which ensures that the source and load conditions are considerably closer to

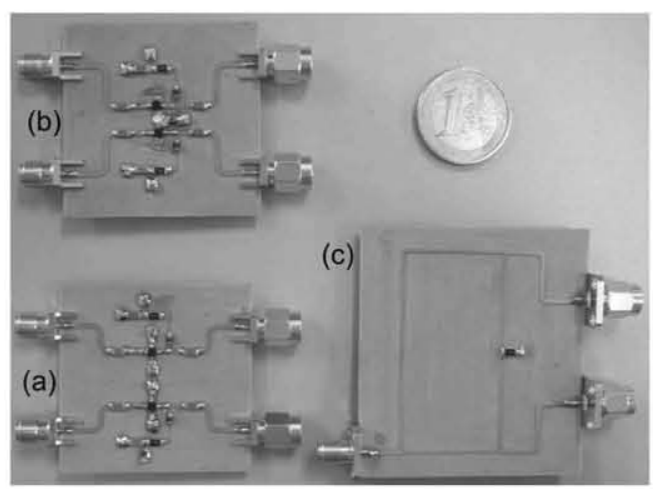

Fig. 4. Photographs of the: (a) balanced and (b) fully differential amplifiers and (c) one of the baluns.

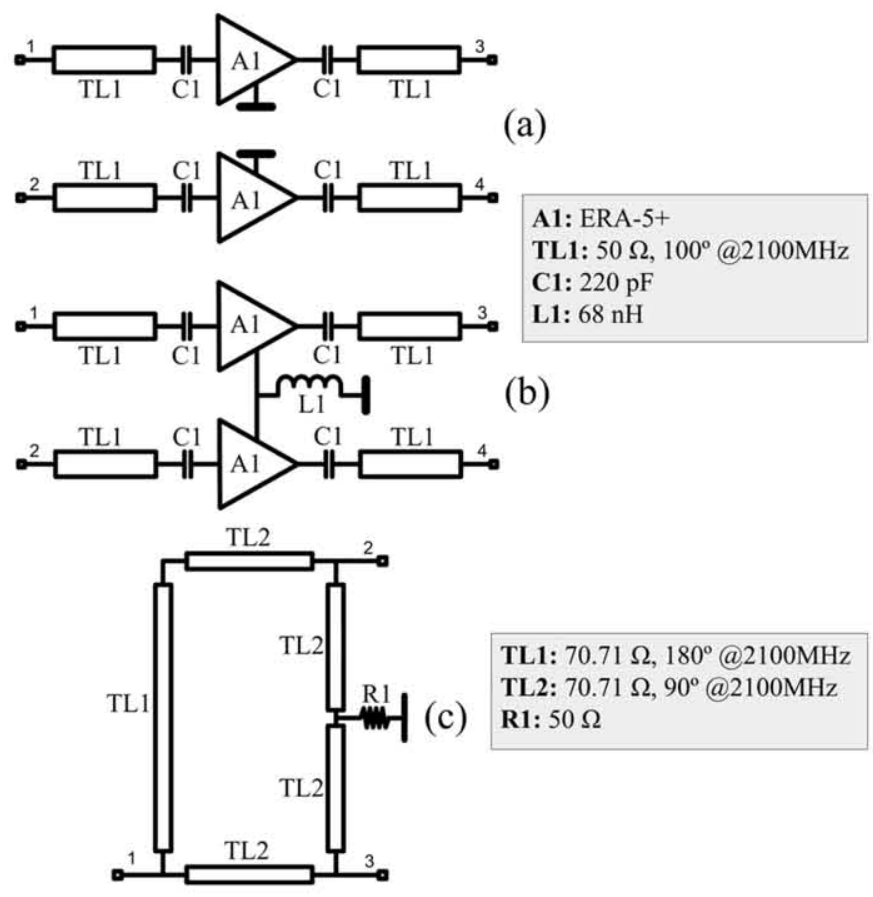

Fig. 5. Circuit schematics of the: (a) balanced and (b) fully differential amplifiers and (c) balun based on a rat-race hybrid.

the desired $50 \Omega$. The active devices have been implemented by using commercial single-ended amplifiers, model ERA-5+ from Minicircuits, Brooklyn, NY. This gain block provides a broadband amplifying device, already matched to $50 \Omega$. Fig. 5 shows the circuit schematic of the manufactured balanced and fully differential amplifiers. The balanced amplifier has been designed just by connecting two independent ERA-5+ amplifiers in parallel [see Fig. 5(a)]. The fully differential amplifier has been implemented using two ERA-5+ amplifiers, but interconnecting the two sources of the amplifiers to ground by means of an intermediate high- $Q$ lumped inductor acting as current source [see Fig. 5(b)]. Capacitors C1 are just dc block devices so their value is chosen to be high to provide low impedance (ideally an open circuit for dc and short circuit for ac). In the case of the inductor L1, it should act as an open circuit for ac and short circuit for dc, which means a high inductance value. However, very high inductance values lead to undesired parasitic resistances in physical inductors, thus a tradeoff value of $68 \mathrm{nH}$ has been chosen. 


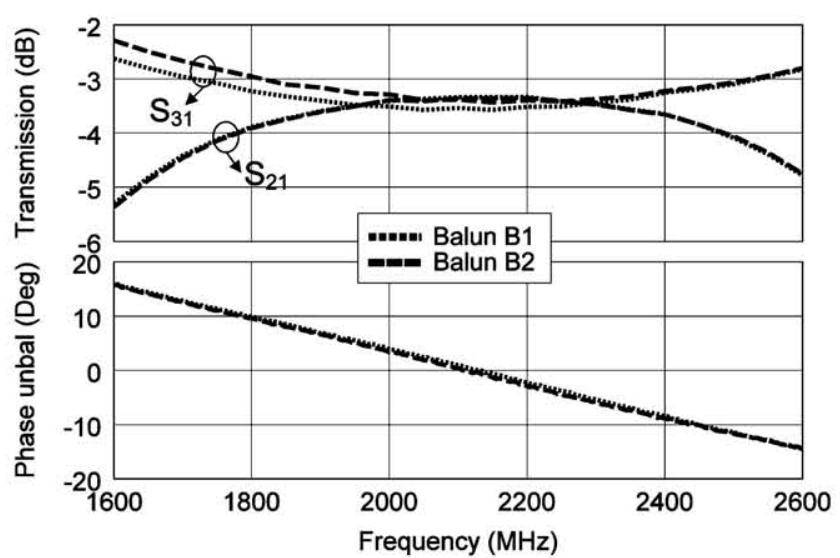

Fig. 6. Measured transmission parameters and phase unbalance of the baluns.

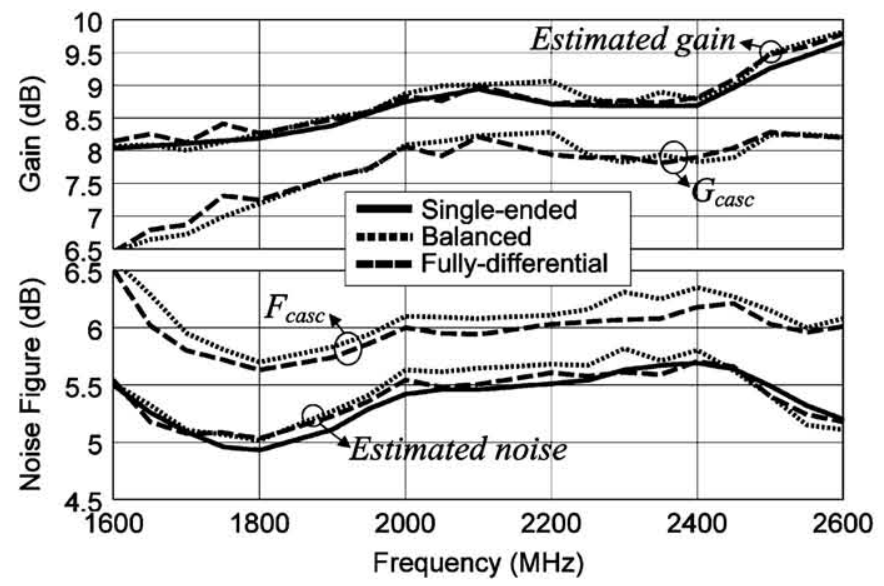

Fig. 7. Measured results with the balanced and fully differential amplifiers, before and after the de-embedding process, and comparison with the performance of the single-ended amplifier.

The measured results for the gain and for the noise have been plotted in Fig. 7. The gain and noise measurements have been obtained using the noise analyzer N8975A from Agilent Technologies, Santa Clara, CA. Two sets of curves have been represented in each graph. The continuous lines represent the gain and noise curves obtained from the single-ended amplifier. A set of lines that are labeled as $G_{\text {casc }}$ and $F_{\text {casc }}$, respectively, represents the measured gain and noise curves directly obtained from the cascaded system formed by the baluns and the corresponding balanced/fully differential amplifier. The actual performance of the differential amplifiers should be the same as the performance of the single-ended amplifier of the two branches (for equivalent loading conditions). Thus, if no de-embedding procedure is applied to the measurement, the gain would be underestimated up to $1.5 \mathrm{~dB}$ and the noise would be overestimated up to $1 \mathrm{~dB}$ with respect to the actual value (i.e., single-ended measurement) in the worst cases. In the edges of the band, the error is higher due to the larger phase and magnitude unbalances in the baluns. In the center of the band, although the unbalances are not significant, the error is still high due to the balun losses. The gain and noise curves after the de-embedding procedures are labeled as "Estimated Gain" and "Estimated Noise" in the graph. Such de-embedded gain and noise curves have been compared with the curves measured from the single-ended amplifier, obtaining a good agreement. It can be seen that both losses

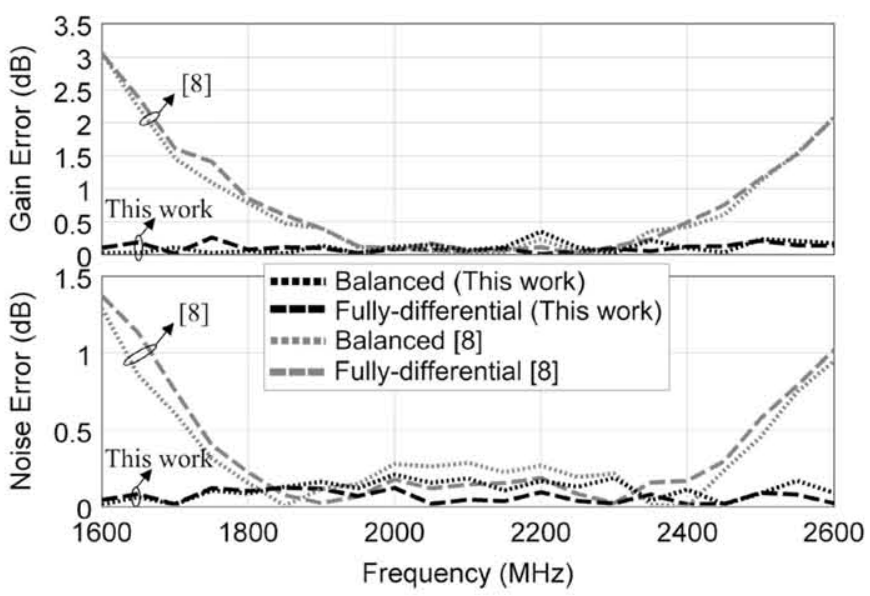

Fig. 8. Measured gain and noise-figure errors obtained with the method proposed in this paper and with the method described in [8] for both differential amplifier implementations.

and phase/magnitude unbalance effects have been significantly corrected following the proposed de-embedding methodology (Section II-C).

It is remarked that other de-embedding procedures do not deal with magnitude and phase unbalances in the baluns. In [8], it is assumed that the outputs of the balun are $180^{\circ}$ out-of-phase and the transmission between ports $1-2$ and $1-3$ are equal in module. Thus, only the value of $\left|s_{21}\right|$ is used to characterize each balun. A comparison between the de-embedding method described in [8] (based on the 1-2 branch of the balun, as specified in [8, Fig. 2]) and the one proposed in this paper is done in Fig. 8. This graph represents the error after the de-embedding the gain and the noise figure of the two differential amplifiers by using the two methods. This error is defined as the difference (in module) between the actual performance of the amplifier (i.e., single-ended measurement) and the performance obtained after the de-embedding procedure. It can be seen that the present method presents much better results, especially at the frequencies in the edge of the band, at which the unbalances in the baluns are more significant. Since the proposed analysis does not only consider the losses in the balun (as in [8]), but also the phase and magnitude unbalances, the present method covers a more general case, and therefore, it is inherently more accurate.

\section{CONCLUSION}

Accurate and simple measurement of differential amplifiers at microwave frequencies is needed for some current telecommunication applications. Using baluns to characterize a differential amplifier allows the use of conventional measurement equipments. However, the effect of the baluns should be properly eliminated. This paper has presented a complete analysis of this measurement setup, using a general model of the baluns with arbitrary losses and unbalances. The propagation of correlated noise signals along the structure, including both common and differential-mode contributions, has been considered. From this analysis, it is possible to exactly extract the desired gain and noise performance of the isolated amplifier. The theory has been validated by means of computer-aided design (CAD) simulations and the measurement of two amplifier prototypes, obtaining good results. Future research can be oriented toward im- 
proving this theoretical study, including a general model of the active device as, for example, assuming arbitrary CMRR values in the amplifier.

\section{REFERENCES}

[1] L. E. Garcia-Muñoz, O. Garcia-Perez, V. Gonzalez-Posadas, J. L. Vazquez-Roy, D. Segovia-Vargas, J. M. Serna-Puente, T. Finn, and J. A. Lopez-Fernandez, "FIDA3: A novel active array design for the midfrequency range of the square kilometre array," in Eur. Antennas Propag. Conf., Barcelona, Spain, Apr. 2010, pp. 1-4.

[2] W. R. Eisenstadt, B. Stengel, and B. M. Thomson, Microwave Differential Circuit Design Using Mixed Mode S-Parameters. Norwood, MA: Artech House, 2006.

[3] D. E. Bockelman and W. R. Eisenstadt, "Combined differential and common-mode scattering parameters: Theory and simulation," IEEE Trans. Microw. Theory Tech., vol. 43, no. 7, pp. 1530-1539, Jul. 1995.

[4] D. E. Bockelman and W. R. Eisenstadt, "Pure-mode network analyzer for on-wafer measurements of mixed-mode $S$-parameters of differential circuits," IEEE Trans. Microw. Theory Tech., vol. 45, no. 7, pp. 1071-1077, Jul. 1997.

[5] J. Morawietz, R. H. Witvers, J. G. B. de Vaate, and E. E. M. Woestenburg, "Noise characterization of ultra low noise differential amplifiers for next generation radiotelescopes," in Eur. Microw. Conf., Munich, Germany, Oct. 2007, pp. 1570-1573.

[6] L. Belostotski and J. W. Haslett, "A technique for differential noise figure measurement of differential LNAs," IEEE Trans. Instrum. Meas., vol. 57 , no. 7, pp. 1298-1303, Jul. 2008.

[7] O. Garcia-Perez, L. E. Garcia-Munoz, V. Gonzalez-Posadas, and D. Segovia-Vargas, "Source-pull characterization of differential active antennas for radio-astronomy applications," in Eur. Wireless Tech. Conf., Rome, Italy, Sep. 2009, pp. 84-87.

[8] A. Abidi and J. C. Leete, "De-embedding the noise figure of differential amplifiers," IEEE J. Solid-State Circuits, vol. 34, no. 6, pp. 882-885, Jun. 1999.

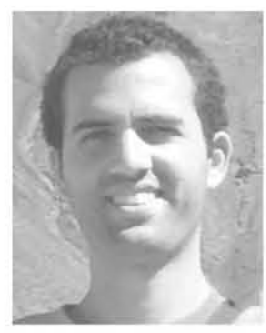

Óscar García-Pérez was born in Madrid, Spain, on December 23, 1984. He received the Engineer degree in telecommunications from Universidad Carlos III de Madrid (UC3M), Madrid, Spain, in 2007, and is currently working toward the Ph.D. degree in communications at UC3M.

His main research interests are the study of microwave active circuits with metamaterial structures and broadband differential low-noise amplifiers for radio astronomy applications.

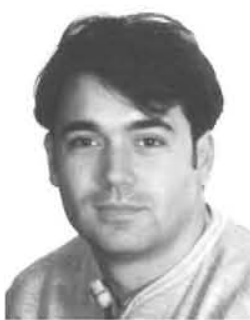

Vicente González-Posadas was born in Madrid, Spain, in 1968. He received the Ing. Técnico degree in radio-communication engineering from the Polytechnic University of Madrid (UPM), Madrid, Spain, in 1992, the M.S. degree in physics from the Universidad Nacional de Educación a Distancia (UNED), Madrid, Spain, in 1995, and the Ph.D. degree in telecommunication engineering from Universidad Carlos III de Madrid (UC3M), Madrid, Spain, in 2001.

$\mathrm{He}$ is currently an Associate Professor with the Technical Telecommunication School, UPM. He has authored or coauthored over 80 technical conference, letter, and journal papers. His interests are related to active antennas, microstrip antennas, composite right/left-handed (CRLH) lines, and metamaterials and microwave technology.

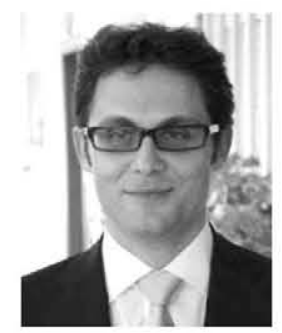

Luis Enrique García-Muñoz is currently an Associate Professor with the Universidad Carlos III de Madrid (UC3M), Madrid, Spain. He has managed or participated in several national and European research projects on areas such as antennas and array design. He has coauthored over 50 papers in international journals and conferences. He holds three patents. His current research interests include terahertz antennas, array design, truncation in antenna arrays, and radio astronomy instrumentation.

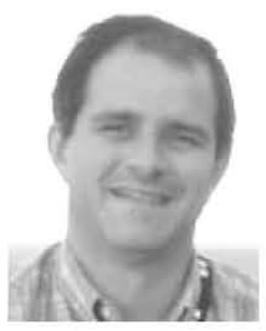

Daniel Segovia-Vargas (M'98) was born in Madrid, Spain, in 1968. He received the Telecommunication Engineering degree and Ph.D. degree from the Polytechnic University of Madrid (UPM), Madrid, Spain, in 1993 and 1998 , respectively.

From 1993 to 1998, he was an Assistant Professor with Valladolid University. Since 1999, he has been an Associate Professor with Universidad Carlos III de Madrid (UC3M), Madrid, Spain, where he is in charge of the microwaves and antenna courses. Since 2004, he has been the leader of the Radiofrequency Group, Department of Signal Theory and Communications, UC3M. He has authored or coauthored over 110 technical conference, letters, and journal papers. His research areas are printed antennas and active radiators and arrays, broadband antennas, left-handed (LH) metamaterials, terahertz antennas, and passive circuits.

Dr. Segovia-Vargas has been and expert of the European Projects Cost260, Cost284, and COST IC0603. 